\title{
A HUREWICZ SPECTRAL SEQUENCE FOR HOMOLOGY
}

\author{
DAVID A. BLANC
}

\begin{abstract}
For any connected space $\mathbf{X}$ and ring $R$, we describe a first-quadrant spectral sequence converging to $\widetilde{H}_{*}(\mathbf{X} ; R)$, whose $E^{2}$-term depends only on the homotopy groups of $\mathbf{X}$ and the action of the primary homotopy operations on them. We show that (for simply connected $\mathbf{X}$ ) the $E^{2}$-term vanishes below a line of slope $1 / 2$; computing part of the $E^{2}$-term just above this line, we find a certain periodicity, which shows, in particular, that this vanishing line is best possible. We also show how the differentials in this spectral sequence can be used to compute certain Toda brackets.
\end{abstract}

\section{INTRODUCTION}

1.1. A Hurewicz spectral sequence. We describe here, for any pointed connected space $\mathbf{X}$ and ring $R$, a first-quadrant spectral sequence, first proposed by H. Miller, which converges strongly to the reduced homology of $\mathbf{X}$ with coefficients in $R$.

1.1.1. The $E^{2}$-term. For $R=\mathrm{Z}$, the $E^{2}$-term of this spectral sequence is isomorphic to the derived functors of a certain "indecomposables" functor on the homotopy algebra of $\mathbf{X}(\S 3.12)$, which takes $\pi_{*} \mathbf{X}$ to the graded group $Q\left(\pi_{*} \mathbf{X}\right)$, defined to be the quotient of $\pi_{*} \mathbf{X}$ by the subgroup of elements which are in the image of a "nontrivial" primary homotopy operation (see $\S 2.2 .1$ below).

1.1.2. The Hurewicz homomorphism. For any space $\mathbf{X}$, the Hurewicz homomorphism $h: \pi_{*} \mathbf{X} \rightarrow \widetilde{H}_{*}(\mathbf{X} ; \mathbf{Z})$ factors through the "indecomposables" $Q\left(\pi_{*} \mathbf{X}\right)$; for $R=\mathbf{Z}$, the edge homomorphism of the spectral sequence, $E_{0, *}^{2} \rightarrow \widetilde{H}_{*}(\mathbf{X} ; \mathbf{Z})$, may be identified with this graded homomorphism $Q\left(\pi_{*} \mathbf{X}\right) \rightarrow \widetilde{H}_{*}(\mathbf{X} ; \mathbf{Z})$ from the "indecomposables" to homology (see $\S 2.2 .3$ below).

1.2. Statement of results. We prove two types of results about the Hurewicz spectral sequence:

1.2.1 Vanishing results. We show that the $E^{2}$-term of the spectral sequence has a vanishing line of slope $1 / 2$, which depends on the connectivity of $\mathbf{X}$ and

Received by the editors June 1, 1988 and, in revised form, July 30, 1988.

1980 Mathematics Subject Classification (1985 Revision). Primary 55T99; Secondary 18G50, $55 \mathrm{~N} 99$.

Key words and phrases. Derived functors, homology, homotopy, Hurewicz homomorphism, ПIalgebras, spectral sequences. 
the coefficients $R$; we also calculate part of the $E^{2}$-term above these lines for any 2-connected space $\mathbf{X}$. More specifically, we show:

(i) If $R$ is any ring and $\mathbf{X}$ is $(r-1)$-connected for $r \geq 3$, then in the Hurewicz spectral sequence for $\mathbf{X}$ with coefficients in $R$ we have $E_{n, k}^{2}=0$ for $n>2(k-r)+1$. If $r=2$, then $E_{n, k}^{2}=0$ for $n>2 k-2$.

(ii) If $\mathbf{X}$ is $(r-1)$-connected $(r \geq 3)$, then $E_{1, r}^{2} \cong \operatorname{Tor}\left(\pi_{r} \mathbf{X}, R\right)$ and $E_{n, k}^{2} \cong$ $\operatorname{Tor}\left(\operatorname{Tor}\left(\pi_{r} \mathbf{X}, \mathbf{Z} / 2\right), R\right)$ for $n=2(k-r)+1 \geq 3$. Taking $R=\mathbf{Z} / 2=\pi_{r} \mathbf{X}$, e.g., we see that the vanishing line of (i) is best possible.

(iii) If $R \subseteq \mathbf{Q}$ (the rationals) and $\mathbf{X}$ is $(r-1)$-connected $(r \geq 3)$, then $E_{n, k}^{2}=0$ for $n>2(k-r)$. If $r=2$, then $E_{n, k}^{2}=0$ for $n>2 k-3$.

(iv) If $R \subseteq \mathbf{Q}$ and $\mathbf{X}$ is $(r-1)$-connected $(r \geq 3)$, then $E_{0, r}^{2} \cong \pi_{r} \mathbf{X} \otimes R$ and $E_{n, k}^{2} \cong \operatorname{Tor}\left(\pi_{r} \mathbf{X}, \mathbf{Z} / 2\right) \otimes R$ for $n=2(k-r) \geq 2$. This shows that the vanishing line of (iii) is also best possible.

1.2.2. Secondary operations. We also illustrate the fact that the differentials in the spectral sequence are related to secondary homotopy information by computing a certain Toda bracket:

For the Moore space $\mathbf{X}=\Sigma^{r-1} \mathbf{R} \mathbf{P}^{2} \quad(r \geq 4)$, we have $\pi_{r} \mathbf{X} \cong \mathbf{Z} / 2$ (with generator $\alpha$ ), and $\pi_{r+2} \mathbf{X} \cong \mathbf{Z} / 4$ (with generator $\beta$ ). We shall make use of a differential in the spectral sequence for $\mathbf{X}$ to give a new proof of the wellknown fact that $\beta$ is in the Toda bracket $\left\langle\alpha, 2, \eta_{r}\right\rangle \subset \pi_{r+2} \mathbf{X}$, where $\eta_{r} \in \pi_{r+1} \mathbf{S}^{r}$ denotes the suspended Hopf map.

Organization. In $\S 2$ we set up the spectral sequence, and identify the edge map with the Hurewicz homomorphism. In $\S 3$ we describe the category of $\Pi$-algebras, recall the definition of derived functors in this context, and identify the $E^{2}$-term of the spectral sequence with the derived functors of "indecomposables." In $\S 4$ we derive the vanishing lines of $1.2(\mathrm{i})$ and (iii), by constructing a suitable free resolution for $\pi_{*} \mathbf{X}$, and in $\S 5$ a calculation shows that these vanishing lines are best possible (as in 1.2(ii) and (iv)). In $\S 6$ a differential in the spectral sequence for $\Sigma^{r-1} \mathbf{R} \mathbf{P}^{2}$ is used to compute the Toda bracket of $\S 1.2 .2$. Finally, in $\S 7$ we mention two related spectral sequences.

\section{ACKNOWLEDGMENTS}

I wish to thank my advisor, Dan Kan, for his help and advice in writing this paper. I also thank Phil Hirschhorn, Chris Stover, and Haynes Miller for many useful conversations.

\section{The SPECTRAL SEQUENCE}

In this section we set up the Hurewicz spectral sequence for any space $\mathbf{X}$ and ring $R(\S 2.1)$ and justify its name by identifying its edge map with the Hurewicz homomorphism $(\S 2.2)$. 
2.1. Setting up the spectral sequence. Let $\mathscr{T}_{*}$ be the category of connected pointed $C W$-complexes; we shall denote the objects of $\mathscr{T}_{*}$ by boldface variables $(\mathbf{X}, \mathbf{Y}, \ldots)$.

2.1.1. Resolution of a space. In [18, §2], C. Stover constructed, for any $\mathbf{X} \in$ $\mathscr{T}_{*}$, a functorial simplicial resolution of $\mathbf{X}$ by wedges of spheres-that is, a simplicial space $V . \mathbf{X}$, together with an augmentation $d_{0}: V_{0} \mathbf{X} \rightarrow \mathbf{X}$, having the following properties:

(a) Each $V_{n} \mathbf{X}$ is a pointed $C W$-complex which is homotopy equivalent to a wedge of spheres.

(b) Each degeneracy map $s_{j}: V_{n} \mathbf{X} \rightarrow V_{n+1} \mathbf{X}$ is an inclusion of $C W$ complexes.

(c) For each $k \geq 1$, the homotopy groups of the simplicial group $\pi_{k} V . \mathbf{X}$ (obtained by applying the functor $\pi_{k}$ dimensionwise to each space in $V . \mathbf{X}$ ) vanish in dimensions $\geq 1$, and the augmentation induces an isomorphism $\pi_{0}\left(\pi_{k} V . \mathbf{X}\right) \cong \pi_{k} \mathbf{X}$.

In particular, this implies that the realization $\Delta V . \mathbf{X}$ of the simplicial space $V$.X (cf. $[17, \S 1]$ ) is homotopy equivalent to $\mathbf{X}$.

2.1.2. A bisimplicial $R$-module. For any ring $R$, let $R S$ denote the functor which assigns to a pointed topological space $\mathbf{X}$ the simplicial $R$-module of its singular chains with coefficients in $R$-that is, the free simplicial $R$-module on the singular complex $S \mathbf{X}$, with basepoint $*=0$. Note that $\pi_{k}(R S \mathbf{X}) \cong$ $\widetilde{H}_{k}(\mathbf{X} ; R)$ for all $k \geq 0[12, \S 2]$.

Applying this dimensionwise to the simplicial space $V . \mathbf{X}$, we get a bisimplicial $R$-module $R S V . \mathbf{X}$; its diagonal $\operatorname{diag}(R S V . \mathbf{X})$ is homotopy equivalent to $R S(\Delta V . \mathbf{X})$ and thus to the simplicial $R$-module $R S \mathbf{X}$.

2.1.3. The spectral sequence. Now we define the Hurewicz spectral sequence for the space $\mathbf{X}$, with coefficients in $R$, to be the Quillen spectral sequence of the bisimplicial $R$-module $R S V . \mathbf{X}$ (see [16]); it has $E_{n, k}^{2} \cong \pi_{n}^{h} \pi_{k}^{v}(R S V . \mathbf{X}$ ) and converges to the associated graded $R$-module of the homotopy of the diagonal: $\pi_{n+k}(\operatorname{diag}(R S V . \mathbf{X}))$. Thus, the Hurewicz spectral sequence converges to the reduced homology of $\mathbf{X}$ with coefficients in $R$, since $\pi_{n+k}(\operatorname{diag}(R S V . \mathbf{X})) \cong$ $\pi_{n+k}(R S \mathbf{X}) \cong \widetilde{H}_{n+k}(\mathbf{X} ; R)$ (by $\left.\S 2.1 .2\right)$.

2.2. Indecomposables and the Hurewicz homomorphism. We now identify the first column of the $E^{2}$-term of this spectral sequence as a functor of $\pi_{*} \mathbf{X}$ and relate it to the Hurewicz homomorphism:

2.2.1. Indecomposables. For any space $\mathbf{X}$, the graded group $\pi_{*} \mathbf{X}=\left\{\pi_{k} \mathbf{X}\right\}_{k=1}^{\infty}$ has a graded subgroup $P\left(\pi_{*} \mathbf{X}\right)$, generated by all elements which are in the image of a nontrivial primary homotopy operation (i.e., any homotopy operation which vanishes in homology). Thus, $P\left(\pi_{*} \mathbf{X}\right)$ is generated by:

(a) compositions $\alpha \circ \zeta \in \pi_{k} \mathbf{X}$, for $\alpha \in \pi_{r} \mathbf{X}$ and $\zeta \in \pi_{k} \mathbf{S}^{r}, k>r>1$; 
(b) Whitehead products (cf. [19, Chapter X, §3])-that is

- elements of the form $[\alpha, \beta] \in \pi_{p+q-1} \mathbf{X}$, for any $\alpha \in \pi_{p} \mathbf{X}$ and $\beta \in \pi_{q} \mathbf{X}, p, q>1$;

- elements of the form $\alpha^{\xi}-\alpha \in \pi_{r} \mathbf{X}$, for $\alpha \in \pi_{r} \mathbf{X} \quad(r>1)$ and $\xi \in \pi_{1} \mathbf{X}$ (where $\alpha^{\xi}$ denotes the result of the action of $\xi$ on $\alpha$ );

- commutators $[\alpha, \beta]=\alpha \beta \alpha^{-1} \beta^{-1} \in \pi_{1} \mathbf{X}$, for $\alpha, \beta \in \pi_{1} \mathbf{X}$.

The quotient graded group $Q\left(\pi_{*} \mathbf{X}\right)=\pi_{*} \mathbf{X} / P\left(\pi_{*} \mathbf{X}\right)$ will be called the indecomposables of $\pi_{*} \mathbf{X}$. Note that $Q\left(\pi_{*} \mathbf{X}\right)$ in degree 1 is simply the Abelianization of $\pi_{1} \mathbf{X}$-so that $Q\left(\pi_{*} \mathbf{X}\right)$ is actually a graded Abelian group.

2.2.2. The column $E_{0, *}^{2}$. For an $n$-sphere $\mathbf{S}^{n}$, the graded group $Q\left(\pi_{*} \mathbf{S}^{n}\right)$ is isomorphic to $\pi_{n} \mathbf{S}^{n} \cong \mathbf{Z}$ in degree $n$ and vanishes in degrees $\neq n$. Thus, if $\mathbf{W}$ has the homotopy type of a wedge of spheres, Hilton's theorem [4, Theorem A] implies that we have an isomorphism of graded Abelian groups: $Q\left(\pi_{*} \mathbf{W}\right) \cong$ $H_{*}(\mathbf{W} ; \mathbf{Z})$.

Combining this with the fact that $V . \mathbf{X}$ is a resolution of $\mathbf{X}$, in the sense of $\S 2.1 .1$, we readily see that the 0 th column of the $E^{2}$-term of the spectral sequence for $\mathbf{X}$ with $R=\mathbf{Z}$ may be identified with the indecomposables of $\pi_{*} \mathbf{X}$.

A similar argument shows that for any ring $R$ and space $\mathbf{X} \in \mathscr{T}_{*}$, in the Hurewicz spectral sequence for $\mathbf{X}$ with coefficients in $R$ we have an isomorphism of graded $R$-modules $E_{0, *}^{2} \cong Q\left(\pi_{*} \mathbf{X}\right) \otimes R$ between the 0 th column of the $E^{2}$-term and the " $R$-indecomposables" of $\pi_{*} \mathbf{X}$.

2.2.3. The Hurewicz homomorphism. For any space $\mathbf{X}$, the Hurewicz homomorphism (considered as a morphism of graded groups $h: \pi_{*} \mathbf{X} \rightarrow \widetilde{H}_{*}(\mathbf{X} ; \mathbf{Z})$ ) vanishes on $P\left(\pi_{*} \mathbf{X}\right) \subset \pi_{*}(\mathbf{X})$, so that it factors through a graded homomorphism $\hat{h}: Q\left(\pi_{*} \mathbf{X}\right) \rightarrow \widetilde{H}_{*}(\mathbf{X} ; \mathbf{Z})$. It may be verified that the edge homomorphism of the integral Hurewicz spectral sequence-that is, the composition $E_{0, *}^{2} \rightarrow$ $E_{0, *}^{\infty} \hookrightarrow \widetilde{H}_{*}(\mathbf{X} ; \mathbf{Z})$-is equal to this homomorphism $\hat{h}: Q\left(\pi_{*} \mathbf{X}\right) \rightarrow \widetilde{H}_{*}(\mathbf{X} ; \mathbf{Z})$ under the identification $E_{0, *}^{2} \cong Q\left(\pi_{*} \mathbf{X}\right)$.

Thus the 0th filtration on $\widetilde{H}_{*}(\mathbf{X} ; \mathbf{Z})$, given by $E_{0, *}^{\infty}$, is precisely the image of the Hurewicz homomorphism-the (graded) subgroup of all spherical elements.

\section{THE $E^{2}$-TERM AS DERIVED FUNCTORS}

We now give a description of the $E^{2}$-term of the Hurewicz spectral sequence in terms of derived functors ( $\$ 3.3)$, after first reviewing the definitions of the category of $\Pi$-algebras $(\S 3.1)$ and of non-Abelian derived functors $(\S 3.2)$.

3.1. $\Pi$-algebras. We recall the definition of the category of $\Pi$-algebras, whose objects are modeled on the homotopy groups of a space, together with the action of all primary homotopy operations on them: 
3.1.1. Definition. Let $h o \mathscr{T}_{*}$ denote the homotopy category of connected pointed $C W$-complexes, and let $\Pi \subset h_{\circ} \mathscr{T}_{*}$ be the full subcategory whose objects are finite wedges of spheres (one for each homotopy type). A П-algebra is then defined to be a contravariant functor $Y: \Pi \rightarrow$ Sets, which takes coproducts to products.

Equivalently, we can think of a $\Pi$-algebra $Y$ as a graded group $\left\{\pi_{j}^{\prime} Y\right\}_{j=1}^{\infty}$ (where we write $\pi_{j}^{\prime} Y$ for $Y\left(\mathbf{S}^{j}\right)$ ), together with an action of the set of primary homotopy operations which satisfies all universal relations on such operations. We denote the category of $\Pi$-algebras by $\Pi$ - $\mathrm{Alg}$.

For any $\Pi$-algebra $Y$ we let $|Y|$ denote the least $k \leq \infty$ for which $\pi_{k}^{\prime} X \neq 0$. If $|Y|>k$, we say that $Y$ is $k$-connected.

3.1.2. Free $\Pi$-algebras. Let $\pi_{*}$ be the functor which assigns to a pointed connected space $\mathbf{X}$ its homotopy $\mathbf{\Pi}$-algebra $\pi_{*} \mathbf{X}$-i.e., $\pi_{*} \mathbf{X}=\left\{\pi_{j} \mathbf{X}\right\}_{j=1}^{\infty}$, with the given action of the homotopy operations. The free $\Pi$-algebras are those which are isomorphic to $\pi_{*} \mathbf{W}$, for some (possibly infinite) wedge of spheres $\mathbf{W} \in \mathscr{T}_{*}$ :

More precisely, let $T$ be a graded set $\left\{T_{j}\right\}_{j=1}^{\infty}$, and let $\mathbf{W}=\bigvee_{j=1}^{\infty} \bigvee_{x \in T_{j}} \mathbf{S}_{x}^{j}$, where each $\mathbf{S}_{x}^{j}$ is a $j$-sphere. Then we say that $\pi_{*} \mathbf{W}$ is the free $\Pi$-algebra generated by $T$. We shall consider each element $x \in T_{j}$ to be an element of $\pi_{*} \mathbf{W}$, by identifying it with that generator of $\pi_{j} \mathbf{W}$ which represents the inclusion $\mathbf{S}_{x}^{j} \hookrightarrow \mathbf{W}$.

3.2. Nonabelian derived functors. We now recall Quillen's definition of derived functors in our context-see [14, part II, §4] and [15, §2]:

3.2.1. Free simplicial $\Pi$-algebras. A simplicial $\Pi$-algebra $A$. is called free iff for each $n \geq 0$ there is a graded set $T^{n} \subseteq A_{n}$ such that $A_{n}$ is the free $\Pi$ algebra generated by $T^{n}$ (§3.1.2), and each degeneracy map $s_{j}: A_{n} \rightarrow A_{n+1}$ takes $T^{n}$ to $T^{n+1}$. The sequence $T^{0}, T^{1}, \ldots$ will be called a set of generators for $A_{\text {. }}$.

3.2.2. Free simplicial resolutions. We define a free simplicial resolution of a $\Pi$ algebra $Y$ to be a free simplicial $\Pi$-algebra $A$, , together with an augmentation $d_{0}: A_{0} \rightarrow Y$, such that for each $k \geq 1$

(a) the homotopy groups of the simplicial group $\pi_{k}^{\prime} \mathbf{A}$. vanish in dimensions $n \geq 1$;

(b) the augmentation induces an isomorphism $\pi_{0}\left(\pi_{k}^{\prime} A\right.$. $) \cong \pi_{k}^{\prime} Y$.

3.2.3. Example. For any space $\mathbf{X} \in \mathscr{T}_{*}$, the resolution $V . \mathbf{X} \rightarrow \mathbf{X}$ of $\S 2.1 .1$ clearly gives rise to a free simplicial resolution $\pi_{*} V \mathbf{X}$ of the $\Pi$-algebra $\pi_{*} \mathbf{X}$.

3.2.4. Definition of derived functors. Let $\mathscr{A}$ be an Abelian category and $T: \Pi$ Alg $\rightarrow \mathscr{A}$ a functor. The $n$th left derived functor of $T$ is the functor $L_{n}(T): \Pi$ $A l g \rightarrow \mathscr{A}$, which assigns to a $\Pi$-algebra $Y$ the object $L_{n}(T) Y \cong \pi_{n}(T A.) \in \mathscr{A}$, where $A . \rightarrow Y$ is any free simplicial resolution of $Y$. (As usual, different resolutions yield equivalent derived functors.) 
3.3. The $E^{2}$-term. For each $\Pi$-algebra $Y$, we have the graded Abelian group of its indecomposables, $Q(Y)=\left\{Q_{k}(Y)\right\}_{k=1}^{\infty}$, defined as in $\S 2.2 .1$. Thus for any ring $R$ we have a functor $Q_{k}(-) \otimes R: \Pi-A l g \rightarrow R$-Mod for every $k \geq 1$.

As noted in $\S 3.2 .3$, for any space $\mathbf{X}$ the free simplicial $\Pi$-algebra $\pi_{*} V . \mathbf{X}$ is a free simplicial resolution of the $\Pi$-algebra $\pi_{*} \mathbf{X}$. On the other hand, by $\S 2.2 .2$ and $\S 2.1 .1(\mathrm{a})$, we know that $\pi_{k}^{v} R S V . \mathbf{X} \cong H_{k}(V . \mathbf{X} ; R)$ is isomorphic to the simplicial $R$-module $Q_{k}\left(\pi_{*} V . \mathbf{X}\right) \otimes R$.

Thus, by Definitions 2.1.3 and 3.2.4, the $k$ th row of the $E^{2}$-term of the Hurewicz spectral sequence for $\mathbf{X}$ with coefficients in $R$ may be identified with the derived functors of $Q_{k}(-) \otimes R$, evaluated on $\pi_{*} \mathbf{X}$ :

$$
E_{n, k}^{2} \cong \pi_{n}^{h} \pi_{k}^{v} R S V . \mathbf{X} \cong \pi_{n}\left(Q_{k}\left(\pi_{*} V \mathbf{X}\right) \otimes R\right) \cong L_{n}\left(Q_{k}(-) \otimes R\right) \pi_{*} \mathbf{X} .
$$

\section{VANISHING RESULTS}

As we have just seen, each row in the $E^{2}$-term of the Hurewicz spectral sequence for a space $\mathbf{X}$ can be identified with certain derived functors evaluated on the $\Pi$-algebra $\pi_{*} \mathbf{X}$. In this section we show that, for 2 -connected $\mathbf{X}$, the $E^{2}$-term has the vanishing lines of $\S 1.2 .1(\mathrm{i})$ and (iii), by showing that the derived functors of $Q_{k}$ vanish beyond a certain point:

4.1. Theorem. Let $k \geq r \geq 3$, and let $Y$ be an $(r-1)$-connected $\Pi$-algebra; then $\left(L_{n} Q_{k}\right) Y=0$ for $n>2(k-r)$. If $k \geq r=2$, then $\left(L_{n} Q_{k}\right) Y=0$ for $n>2 k-3$.

Applying the universal coefficients theorem for homology, we obtain the following

4.1.1. Corollary. For $k \geq r$, and $Y$ as above, we have

(i) For any ring $R$,

$$
L_{n}\left(Q_{k}(-) \otimes R\right) Y=0 \quad \text { for } \begin{cases}n>2(k-r)+1 & \text { if } r \geq 3, \\ n>2 k-2 & \text { if } r=2 .\end{cases}
$$

(ii) If $R \subseteq \mathbf{Q}$, then

$$
L_{n}\left(Q_{k}(-) \otimes R\right) Y=0 \quad \text { for } \begin{cases}n>2(k-r) & \text { if } r \geq 3, \\ n>2 k-1 & \text { if } r=2 .\end{cases}
$$

Theorem 4.1 is proved in $\S 4.2$, using a certain resolution $A . \rightarrow Y$ described in Proposition 4.2.2 below. After some remarks on constructing resolutions in $\S 4.3$, the proof of the proposition is outlined in $\S 4.4$ and completed in $\S 4.5$.

4.2. Proof of Theorem 4.1. In order to calculate the derived functors of $Q_{k}$ evaluated on $Y$, any free simplicial resolution $A . \rightarrow Y$ can be used; we wish to describe a particular one, for which we need the following definitions:

4.2.1. Basic ПI-algebras. Given a free simplicial $\Pi$-algebra $A$. and a set of generators $T^{0}, T^{1}, \ldots$ as in $\S 3.2 .1$, we define the $n$th basic $\Pi$-algebra for 
$A_{\text {. }}$, denoted $\bar{A}_{n}$, to be the sub-free $\Pi$-algebra of $A_{n}$ generated by the nondegenerate elements in $T^{n}$.

A sequence $\bar{A}_{0}, \bar{A}_{1}, \ldots, \bar{A}_{n}, \ldots$ of basic $\Pi$-algebras for a free simplicial $\Pi$ algebra $A$. is called a $C W$-basis for $A$. (cf. $[7, \S 5.1]$ ) iff for each $n \geq 0$ we have $\left.d_{j}\right|_{\bar{A}_{n}}=0$ for $1 \leq j \leq n$. We call the morphism $\bar{d}_{0}=\left.d_{0}\right|_{\bar{A}_{n+1}}: \bar{A}_{n+1} \rightarrow A_{n}$ the attaching map for $\bar{A}_{n+1}$. With these definitions we have the following

4.2.2. Proposition. Let $Y$ be an $(r-1)$-connected $\Pi$-algebra $(r \geq 2)$; then $Y$ has a free simplicial resolution $A . \rightarrow Y$, with a $C W$-basis $\bar{A}_{0}, \bar{A}_{1}, \ldots$, such that

(a) for each $n \geq 0$ we have $n \leq 2 \cdot\left(\left|\bar{A}_{n}\right|-r\right)+1$ if $r \geq 3$, and $n \leq 2\left|\bar{A}_{n}\right|-2$ if $r=2$;

(b) for $n=2(k-r)+1$, if $r \geq 3$ (respectively $n=2 k-2$, if $r=2$ ), the attaching map $\bar{d}_{0}: \bar{A}_{n} \rightarrow \bar{A}_{n-1}$, in degree $k$, is a monomorphism into $\pi_{k}^{\prime} \bar{A}_{n-1}$.

4.2.3. Proof of the theorem. Let $Y$ be an $(r-1)$-connected $\Pi$-algebra $(r \geq 2)$, and for $k \geq r$ let $N=2(k-r)+1$ if $r \geq 3$ (or $N=2 k-2$ if $r=2$ ).

(I) Take $A . \rightarrow Y$ to be the resolution of Proposition 4.2.2, and let $B$. $=$ $Q_{k} A$.; by Definition 3.2.4 we have $\left(L_{n} Q_{k}\right) Y \cong \pi_{n} B$. . Now recall that for a simplicial Abelian group such as $B$., we have the associated normalized chaincomplex $\left\{N B_{*}, \partial\right\}$, where for each $n \geq 0$ we let

$$
N B_{n}=\bigcap_{1 \leq j \leq n} \operatorname{ker}\left\{d_{j}: B_{n} \rightarrow B_{n-1}\right\} \subset B_{n}, \quad \text { and } \quad \partial_{n}=\left.d_{0}\right|_{N B_{n}} ;
$$

then $\pi_{n} B . \cong H_{n}\left(N B_{*}\right) \quad($ cf. $[12, \S 17])$.

Moreover, if $D B_{n}$ denotes the subgroup of $B_{n}$ generated by the degenerate elements, we have $N B_{n} \cap D B_{n}=0$ (cf. [12, Corollary 22.2]).

(II) In the free simplicial II-algebra $A$. we can write each $A_{n}$ as a coproduct of $\bar{A}_{n}$ with the images of the $\bar{A}_{m}$ 's (for $0 \leq m<n$ ) under various degeneracy maps (see $\S 4.5 .1$ below). However, the functor $Q_{k}$, when restricted to the subcategory of free $\Pi$-algebras, clearly preserves coproducts; also, it vanishes on any $k$-connected $\Pi$-algebra. Since $k<\left|\bar{A}_{n}\right|$ for $n>N=2(k-r)+1$ by Proposition 4.2.2(a), we have $B_{n}=D B_{n}$, so that (I) implies $N B_{n}=0$, and thus $\left(L_{n} Q_{k}\right) Y \cong \pi_{n} B \cong H_{n}\left(N B_{*}\right)=0$ for $n>N$.

(III) Note that if $f: X \rightarrow Y$ is a map between two $(k-1)$-connected free $\Pi$-algebras which is a monomorphism in degree $k(k \geq 2)$, then $Q_{k} f$ is a monomorphism, too (cf. $\S 2.2 .2$ ). Now for $\bar{B}=Q_{k} \bar{A}_{N}$ we have $B_{n} \cong \bar{B} \oplus D B_{N}$. However, by 4.2.2(b) $\bar{d}_{0}: \bar{A}_{N} \rightarrow A_{N-1}$ is a monomorphism in degree $k$ into the $(k-1)$-connected free $\Pi$-algebra $\bar{A}_{N-1}$; therefore, $Q_{k}\left(\left.\bar{d}_{0}\right|_{\bar{A}_{N}}\right)=\left.\left(Q_{k} d_{0}\right)\right|_{\bar{B}}$ is a monomorphism. Thus by $(\mathrm{I})$ we see that $B$. has no $N$-cycles, so that $\left(L_{N} Q_{k}\right) Y \cong \pi_{N} B .=0$.

4.3. Constructing free simplicial resolutions. To prove Proposition 4.2.2, we wish to construct a suitable free simplicial resolution $A . \rightarrow Y$. Now a free 
simplicial $\Pi$-algebra $A$. with $C W$-basis $\bar{A}_{0}, \bar{A}_{1}, \ldots$ may be constructed by a process analogous to that of attaching cells in building up a $C W$-complex:

4.3.1. Normalized cycles. For any simplicial $\Pi$-algebra $A$., we have the usual normalization process, which yields chains and cycles (as defined in [13] for simplicial groups): in particular, the $n$-cycles $\Pi$-algebra of $A$. is the sub- $\Pi$ algebra of $A_{n}$ defined by

$$
Z A_{n}=\bigcap_{0 \leq j \leq n} \operatorname{ker}\left\{d_{j}: A_{n} \rightarrow A_{n-1}\right\} .
$$

4.3.2. $C W$-construction. By analogy with $C W$-complexes, we can construct a free simplicial $\Pi$-algebra by an inductive process, in which we assume we are given an free simplicial $\Pi$-algebra $A$, and obtain a new free simplicial II-algebra $A^{\prime}$. by "attaching" a free $\Pi$-algebra $\bar{A}$ in dimension $n$, by means of an attaching map $\bar{d}_{0}: \bar{A} \rightarrow A_{n}$ (cf. $[8, \S 3]$ ).

For the simplicial identities to hold in the simplicial $\Pi$-algebra $A^{\prime}$. so obtained, we require that $\bar{d}_{0}$ satisfy $d_{j} \circ \bar{d}_{0}=0$ for $0 \leq j \leq n$-that is, that $\bar{d}_{0}: \bar{A} \rightarrow A_{n}$ factor through $Z A_{n} \hookrightarrow A_{n}$. Note that a free simplicial $\Pi$-algebra is completely determined by specifying a $C W$-basis, together with the attaching maps.

4.3.3. Identifying resolutions. In particular, one can show that an free simplicial $\Pi$-algebra $A$., with a $C W$-basis $\bar{A}_{0}, \bar{A}_{1}, \ldots$ and an augmentation $A_{0} \rightarrow$ $Y$, is a free simplicial resolution of $Y$ (Definition 3.2.2) iff for all $n \geq-1$, the attaching map $\bar{d}_{0}: \bar{A}_{n+1} \rightarrow A_{n}$ factors through an epimorphism $\bar{d}_{0}: \bar{A}_{n+1} \rightarrow$ $Z A_{n}$ (where we set $Z A_{-1}=A_{-1}=Y$ ).

4.3.4. $N$-resolutions. If condition 4.3.3 is satisfied only for $0 \leq n<N$, we call $A . \rightarrow Y$ an $N$-resolution. As in $\S 3.2 .2$, this is equivalent to requiring that for each $k \geq 1$, the simplicial group $\pi_{k}^{\prime} A$. has homotopy groups $\pi_{j}\left(\pi_{k}^{\prime} A\right.$.) $=0$ for $1 \leq j<N$, and that the augmentation induces an isomorphism $\pi_{0}\left(\pi_{k}^{\prime} A\right.$. $) \cong$ $\pi_{k}^{\prime} Y$.

4.4. Construction of the resolution. Given an $(r-1)$-connected $\Pi$ I-algebra $Y$ $(r \geq 2)$, we now construct a free simplicial resolution $A . \rightarrow Y$, along with a given $C W$-basis $\bar{A}_{0}, \bar{A}_{1}, \ldots$, as required in Proposition 4.2.2. For simplicity suppose first that $r \geq 3$.

4.4.1. An inductive construction. The free simplicial $\Pi$-algebra $A$. is constructed by induction on $n \geq-1$. At the $n$th stage we assume we have an augmented free simplicial $\Pi$-algebra $A^{n} \rightarrow Y$, with $C W$-basis $\bar{A}_{0}, \bar{A}_{1}, \ldots, \bar{A}_{n}, 0$, $\ldots$, satisfying the following hypotheses:

(i) $A^{n}$. is an $n$-resolution of $Y$ (Definition 4.3.4);

(ii) for each $m \geq 0$ we have $m \leq 2 \cdot\left(\left|\bar{A}_{m}\right|-r\right)+1$;

(iii) for $m=2(k-r)+1 \leq n$ the attaching map $\bar{d}_{0}: \bar{A}_{m} \rightarrow A_{m-1}^{m-1}$, in degree $k$, is a monomorphism into $\pi_{k}^{\prime} \bar{A}_{m-1}$. 
$A^{n+1}$ is then constructed as in $\S 4.3 .2$ by suitable choice of the free $\Pi$-algebra $\bar{A}_{n+1}$ and attaching map $\bar{d}_{0}: \bar{A}_{n+1} \rightarrow A_{n}^{n}$. We start the induction with $Z A_{-1}^{-1}=$ $A_{-1}^{-1}=Y$ and $A_{.}^{-1}=0$.

Finally, set $A .=\bigcup_{n=0}^{\infty} A^{n}$; the induction hypotheses clearly ensure that $A$. will satisfy the requirements of Proposition 4.2.2. Note that we have $A_{n}=A_{n}^{m}$ for $m \geq n$; in particular, we shall write $A_{n}$ for $A_{n}^{n}$. In fact, $A^{n}$. will just be the $n$-skeleton, in the usual sense, of $A$. .

4.4.2. The inductive condition. By $\S 4.3 .4$, all we need in order for $A_{\cdot}^{n+1}$ so constructed to be an $(n+1)$-resolution is that the attaching map $\bar{d}_{0}: \bar{A}_{n+1} \rightarrow$ $Z A_{n}^{n}=Z A_{n}$ be an epimorphism. Thus we may choose $\bar{A}_{n+1}$ so that $\left|\bar{A}_{n+1}\right|=$ $\left|Z A_{n}^{\prime}\right|$.

Therefore, in order to get an $A_{.}^{n+1}$ satisfying the requirements 4.4.1(i) and (ii), it suffices to show that the following holds:

$$
n \leq 2 \cdot\left(\left|Z A_{n}\right|-r\right)
$$

(since we can then let $\bar{A}_{n+1}$ be the free $\Pi$-algebra on the underlying set of $Z A_{n}$, with the obvious attaching map).

Thus we are interested in the connectivity of the $n$-cycles $\Pi$-algebra. Now for each $n \geq 0$, let $Z \bar{A}_{n}=\bar{A}_{n} \cap Z A_{n}=\operatorname{Ker}\left\{\bar{d}_{0}: \bar{A}_{n} \rightarrow A_{n-1}\right\}$; it turns out that these "cycles with nondegenerate support" have the following property:

4.4.3. Lemma. Let $r \geq 3$, and let $A$. be a free simplicial $\Pi$-algebra with $C W$ basis $\bar{A}_{0}, \bar{A}_{1}, \ldots$, such that for each $m \geq 0$ we have $m \leq 2 \cdot\left(\left|\bar{A}_{m}\right|-r\right)+1$ (as in 4.4.1(ii)). Then for $n \geq 2(t-r) \geq 0$ we have $\pi_{t}^{\prime} Z \bar{A}_{n}=\pi_{t}^{\prime} Z A_{n}$.

4.4.4. The two-step construction. Given this lemma, we can now describe the construction of $A$, as required in Proposition 4.2.2, proceeding two steps at a time:

Let $n=2 m-1$ and assume the augmented free simplicial II-algebra $A^{n} \rightarrow$ $Y$ has been chosen, satisfying condition (1) for $n$, as well as the induction hypotheses of $\S 4.4 .1$.

(I) The first step is immediate, given (1): we can obviously choose an $(m+$ $r-1)$-connected free $\Pi$-algebra $\bar{A}_{n+1}$ which has an epimorphism $\bar{d}_{0}: \bar{A}_{n+1} \rightarrow$ $Z A_{n}$, as in $\S 4.4 .2$-and so obtain the free simplicial ח-algebra $A_{.}^{n+1}$, satisfying $\S 4.4 .1(\mathrm{i})-(\mathrm{ii})$.

(II) Note that by Definition 3.1.2, $\bar{A}_{n+1} \cong \pi_{*}\left(\bigvee_{j=m+r}^{\infty} \bigvee_{T_{j}} \mathrm{~S}^{j}\right)$ for some graded set $T$, so that $\pi_{m+r}^{\prime} \bar{A}_{n+1}$ is isomorphic to the free Abelian group generated by $T_{m+r}$. Now consider the II-algebra $Z A_{n+1}$. Using Lemma 4.4.3, we see that $\pi_{m+r}^{\prime} Z A_{n+1}=\pi_{m+r}^{\prime} Z \bar{A}_{n+1} \subseteq \pi_{m+r}^{\prime} \bar{A}_{n+1}$ is free Abelian. This allows us to choose $\bar{A}_{n+2}$ as follows:

Let $K$ be a basis for the free Abelian group $\pi_{m+r}^{\prime} Z A_{n+1}$, and let $B$ be the free $\Pi$-algebra generated by the graded set $S$, where $S_{m+r}=K$ and $S_{i}=\varnothing$ 
for $i \neq m+r$ (cf. §3.1.2). There is an obvious map $f: B \rightarrow Z A_{n+1}$, with $\pi_{m+r}^{\prime} f: \pi_{m+r}^{\prime} B \rightarrow \pi_{m+r}^{\prime} Z A_{n+1}$ an isomorphism.

Let $Z^{\prime}$ denote the $(m+r)$-connected $\Pi$-algebra obtained from $Z A_{n+1}$ by setting $\pi_{m+r}^{\prime} Z A_{n+1}$ equal to 0 ; choose an $(m+r)$-connected free $\Pi$-algebra $C$ which has an epimorphism $g: C \rightarrow Z^{\prime}$, as in $\S 4.4 .2$.

Now let $\bar{A}_{n+2}=B \amalg C$; we have a morphism $\bar{d}_{0}: \bar{A}_{n+2} \rightarrow Z A_{n+1}$ induced by $f$ and $g$; this is an epimorphism, since $\pi_{j}^{\prime} \bar{d}_{0}$ is surjective for each $j \geq$ 1. Moreover, since $\pi_{m+r}^{\prime} \bar{d}_{0}: \pi_{m+r}^{\prime} \bar{A}_{n+2} \rightarrow A_{n+1}$ is an isomorphism, we have $0=\pi_{m+r}^{\prime} Z \bar{A}_{n+2}=\pi_{m+r}^{\prime \prime} Z A_{n+2}$ (using Lemma 4.4.3), so that hypotheses of $\S 4.4 .1(\mathrm{i})$-(iii) hold for $n+2$, too.

4.5. Proof of Lemma 4.4.3. The remainder of this section is devoted to the proof of Lemma 4.4.3. We first need the following

4.5.1. Explicit description. If $A$. is a free simplicial $\Pi$-algebra with $C W$-basis $\bar{A}_{0}, \bar{A}_{1}, \ldots$, then each $A_{n}$ can be described explicitly as a coproduct of basic $\Pi$-algebras, as follows (compare [12, p. 95(i)]):

For each $n \geq 0$ and $0 \leq \lambda \leq n$, let $\mathscr{F}_{\lambda, n}$ denote the set of all sequences of $\lambda$ nonnegative integers $i_{1}<i_{2}<\cdots<i_{\lambda},\left(i_{\lambda}<n\right)$, with $s_{I}=s_{i_{\lambda}} \circ \cdots s_{i_{2}} \circ s_{i_{1}}$ the corresponding $\lambda$-fold degeneracy. (We allow $\lambda=0$, with the corresponding $s_{I}=i d$.) Then

$$
A_{n} \cong \coprod_{0 \leq \lambda \leq n} \coprod_{I \in \mathcal{J}_{\lambda, n}} \bar{A}_{n-\lambda}
$$

where for $I \in \mathscr{I}_{\lambda, n}$, the copy of $\bar{A}_{n-\lambda}$ indexed by $I$ is in the image of the $\lambda$-fold degeneracy $s_{I}$, in the obvious sense.

Thus given $A$. as above, for each $n \geq 0$ we can write: $A_{n} \cong \amalg_{\alpha \in K_{n}} X_{\alpha}$, where each $X_{\alpha}$ is in the image of some $\lambda_{\alpha}$-fold degeneracy $\left(\lambda_{\alpha} \geq 0\right)$. In this situation we have the following

4.5.2. Lemma. Assume that $\coprod_{i=1}^{q} X_{\alpha_{i}}$ is a subcoproduct of the above $A_{n} \cong$ $\amalg_{\alpha \in K_{n}} X_{\alpha}$, which satisfies

$$
\sum_{i=1}^{q}\left(n-\lambda_{\alpha_{i}}\right)<n
$$

Then for some $0 \leq j<n$, each $X_{\alpha_{i}}(1 \leq i \leq q)$ is in the image of $s_{j}$.

4.5.3. Proof. Let $M=\left(m_{i j}\right)$ be the $q \times n$ matrix with

$$
m_{i j}= \begin{cases}0 & \text { if } X_{\alpha_{i}} \text { is in the image of } s_{j}, \\ 1 & \text { otherwise. }\end{cases}
$$

Then each $X_{\alpha_{i}}$ is in the image of at least $\lambda_{\alpha_{i}}$ of the $n$ possible degeneracy maps $s_{j}: A_{n-1} \rightarrow A_{n}$, so there are at most $\left(n-\lambda_{\alpha_{t}}\right)$ entries of 1 in the $i$ th row of $M$. But then condition (3) implies there is some column of 0 's in $M$-i.e., that all the $X_{\alpha_{i}}$ 's have some common direction of degeneracy. 
4.5.4. Completion of proof. We now apply Lemma 4.5.2 to prove Lemma 4.4.3:

One can use (2) of $\S 4.5 .1$ to write $A_{n}$ as a coproduct of copies of the first $n+1$ basic $\Pi$-algebras of $A .: A_{n} \cong \coprod_{0 \leq \lambda \leq n} \coprod_{I \in \mathcal{I}_{\lambda, n}} \bar{A}_{n-\lambda}$.

By hypothesis 4.4.1(ii), for each $\lambda$ we have

$$
(n-\lambda)+2 r-1 \leq 2 \cdot\left|\bar{A}_{n-\lambda}\right| .
$$

Using Hilton's theorem [4, Theorem A] applied to the coproduct (2), we have a direct-sum decomposition of $\pi_{t}^{\prime} A_{n}$, so that any $\gamma \in \pi_{t}^{\prime} Z A_{n} \subset \pi_{t}^{\prime} A_{n}$ can be written as a sum $\gamma=\sum \gamma_{k}$, with each $\gamma_{k}$ an element of some summand in this decomposition:

That is, $\gamma_{k} \in \pi_{t}^{\prime}\left(\bar{A}_{n-\lambda_{1}} \amalg \cdots \amalg \bar{A}_{n-\lambda_{q}}\right)$, where each $\bar{A}_{n-\lambda_{i}}$ is one of the coproduct-summands of $(2)$ and thus is in the image of some $\lambda_{i}$-fold degeneracy $\left(\lambda_{i} \geq\right.$ $0)$. Moreover, we know that

$$
\sum_{i=1}^{q}\left(\left|\bar{A}_{n-\lambda_{i}}\right|-1\right) \leq t-1, \quad \text { where } 2 t \leq n+2 r .
$$

Combining (4) and (5), we find that if $q \geq 2$ we have

$$
\sum_{i=1}^{q}\left(n-\lambda_{i}\right)<n
$$

(since $r \geq 3$ ), so that by Lemma 4.5.3, $\gamma_{k}$ is degenerate. Therefore, every nondegenerate $\gamma_{k}$-for which necessarily $q=1$-is in $\pi_{t}^{\prime} \bar{A}_{n}$, and so is itself an $n$-chain (by the definition of a $C W$-basis- $\$ 4.2 .1$ ). Since $\gamma \in Z \pi_{t}^{\prime} A_{n}$ is in particular an $n$-chain, this implies that the sum of the degenerate $\gamma_{k}$ 's is also an $n$-chain.

However, $t \geq r \geq 2$, so that $B$. $=\pi_{t}^{\prime} A$. is a simplicial Abelian group and $N B_{n} \cap D B_{n}=0$ (see 4.2.3(I)). Thus the sum of the degenerate $\gamma_{k}$ 's must vanish, and we can assume $q=1, \lambda_{1}=0$ for each summand $\gamma_{k}$ in $\gamma$. This implies that $\gamma \in \pi_{t}^{\prime} \bar{A}_{n}$, as required.

With the obvious modifications in Lemma 4.4 .3 for the case $r=2$, this completes the proof of Proposition 4.2.2.

\section{A calculation}

In this section we calculate the derived functors of the functors $Q_{k}$ of $\S 3.3$ just above the vanishing lines of Theorem 4.1, to show:

5.1. Theorem. For $r \geq 3$, let $Y$ be an $(r-1)$-connected $\Pi$-algebra. Then $\left(L_{0} Q_{r}\right) Y \cong \pi_{r}^{\prime} Y$, and for $n=2(k-r) \geq 2$ we have $\left(L_{n} Q_{k}\right) Y \cong \operatorname{Tor}\left(\pi_{r}^{\prime} Y, \mathbf{Z} / 2\right)$. 
Again, by the universal coefficients theorem we have the following:

5.1.1. Corollary. Let $r$ and $Y$ be as above; then

(i) for any ring $R$, we have $L_{1}\left(Q_{r}(-) \otimes R\right) Y \cong \operatorname{Tor}\left(\pi_{r}^{\prime} Y, R\right)$, and if $n=$ $2(k-r)+1 \geq 3$, we have

$$
L_{n}\left(Q_{k}(-) \otimes R\right) Y \cong \operatorname{Tor}\left(\operatorname{Tor}\left(\pi_{r}^{\prime} Y, \mathbf{Z} / 2\right), R\right) .
$$

(ii) If $R \subseteq Q$, we have $L_{0}\left(Q_{r}(-) \otimes R\right) Y \cong \pi_{r}^{\prime} Y \otimes R$, and if $n=2(k-r) \geq 2$, we have

$$
L_{n}\left(Q_{k}(-) \otimes R\right) Y \cong \operatorname{Tor}\left(\pi_{r}^{\prime} Y, \mathbf{Z} / 2\right) \otimes R .
$$

This shows that the vanishing results of Corollary 4.1.1 are the best possible for these rings-at least for 2-connected $\Pi$-algebras-as claimed in $\S 1$-2(ii) and (iv). (Note also that part (i) of the corollary, together with 4.1.1(i), implies 4.1.1(ii) for $r \geq 3$.)

The theorem is proved in $\S 5.2$, using an explicit construction of a free simplicial resolution in $\S 5.3$.

5.2. Proof of Theorem 5.1. In order to calculate the derived functors, we need the following partial description of a specific resolution for $Y$ :

5.2.1. Proposition. Let $Y$ be an $(r-1)$-connected $\Pi$-algebra $(r \geq 3)$, and let

$$
0 \rightarrow H \stackrel{i}{\rightarrow} G \rightarrow \pi_{r}^{\prime} Y \rightarrow 0 \quad \text { and } \quad 0 \rightarrow L \stackrel{j}{\rightarrow} K \rightarrow \operatorname{Tor}\left(\pi_{r}^{\prime} Y, \mathbf{Z} / 2\right) \rightarrow 0
$$

be presentations of the respective Abelian groups (with $G, H, K, L$ free Abelian). There there are a free simplicial resolution $A . \rightarrow Y$ with $C W$ basis $\bar{A}_{0}, \bar{A}_{1}, \ldots$ and free $\Pi$-algebras $\bar{B}_{m}$ and $\bar{C}_{m}$ for each $m \geq 0$ such that $\bar{A}_{m}=\bar{B}_{m} \amalg \bar{C}_{m}$ and

(i) for each $k \geq r$ and $m=2(k-r)$, the free $\Pi$-algebras $\bar{B}_{m}$ and $\bar{B}_{m+1}$ are generated by graded sets concentrated in degree $k$-that is, each is the homotopy II-algebra of a wedge of $k$-spheres;

(ii) the attaching map $\left.\bar{d}_{0}\right|_{\bar{B}_{m+1}}$ factors through $\bar{B}_{m} \hookrightarrow A_{m}$ for each $m \geq 0$;

(iii) there are isomorphisms $f: \pi_{r}^{\prime} \bar{B}_{0} \cong F$ and $g: \pi_{r}^{\prime} \bar{B}_{1} \cong H$ such that $f \circ \pi_{r}^{\prime} \bar{d}_{0}=i \circ g$

(iv) for each $m=2(k-r)>0$, there are isomorphisms $f: \pi_{k}^{\prime} \bar{B}_{m} \cong K$ and $g: \pi_{k}^{\prime} \bar{B}_{m+1} \cong L$ such that $f \circ \pi_{k}^{\prime}\left(\left.\bar{d}_{0}\right|_{\bar{B}_{m+1}}\right)=j \circ g$;

(v) $r+1 \leq\left|\bar{C}_{0}\right|$, and for each $m>0$, we have $m \leq 2 \cdot\left(\left|\bar{C}_{m}\right|-r\right)$;

(vi) for $m=2(k-r)-1>1$, the attaching map $\left.\bar{d}_{0}\right|_{\bar{C}_{m+1}}$, when projected onto $\bar{C}_{m}$, is a monomorphism in degree $k$.

5.2.2. Proof of the Theorem. Let $A . \rightarrow Y$ be the resolution of Proposition 5.2.1. By 5.2.1(iii) and (v), it is clear that

$$
\left(L_{0} Q_{k}\right) Y \cong \pi_{0} Q_{k} A . \cong \operatorname{Cok}\{i: H \rightarrow G\} \cong \pi_{r}^{\prime} Y .
$$


To calculate $\left(L_{n} Q_{k}\right) Y \cong \pi_{n} Q_{k} A$. for $n=2(k-r) \geq 2$, by 5.2 .1 (ii) and (v) we need only consider the " $k$-spheres" of

$$
\bar{B}_{n+1} \stackrel{\bar{d}_{0}}{\rightarrow} \bar{B}_{n} \hookrightarrow \bar{B}_{n} \amalg \bar{C}_{n}=\bar{A}_{n} \stackrel{\bar{d}_{0}}{\rightarrow} A_{n-1} \cdot
$$

However, by 5.2.1(vi), $Q_{k}\left(\left.\bar{d}_{0}\right|_{\bar{C}_{n}}\right)$ is a monomorphism, as in $\S 4.2 .3$ (III), while $\left.\bar{d}_{0}\right|_{\bar{B}_{n}}$ factors through $\bar{B}_{n-1}$ by 5.2 .1 (ii), so that $Q_{k}\left(\left.\bar{d}_{0}\right|_{\bar{B}_{n}}\right)=0$ by $5.2 .1(\mathrm{i})$.

Thus we need only consider $\bar{B}_{n+1} \rightarrow \bar{B}_{n} \rightarrow 0$ in degree $k$, and by 5.2.1(iv) and $\S 2.2 .2$ we therefore have

$$
\pi_{n} Q_{k} A . \cong \operatorname{Cok}\{j: K \rightarrow L\} \cong \operatorname{Tor}\left(\pi_{r}^{\prime} Y, \mathbf{Z} / 2\right) .
$$

5.3. Proof of Proposition 5.2.1. Given an $(r-1)$-connected $\Pi$-algebra $Y$, with $r \geq 3$, the resolution $A$. $\rightarrow Y$ described in Proposition 5.2.1 is constructed (as in $\S 4.4)$ by induction on the skeleta, two steps at a time:

5.3.1. Beginning the induction. We start the induction with $k=r$, choosing $\bar{B}_{0}, \bar{B}_{1}$ to satisfy 5.2.1(i) and (iii). Because $r \geq 3$, by the argument of $\S 4.5 .4$ we can also choose $\bar{C}_{0}, \bar{C}_{1}$ satisfying $5.2 .1(\mathrm{v})$, with the free simplicial $\Pi$-algebra obtained at this stage being a 1 -resolution. Since $r \geq 3$, we have $\pi_{r+1} S^{r} \cong \mathbf{Z} / 2$; thus

$$
\begin{aligned}
\pi_{r+1}^{\prime} Z \bar{B}_{1} & =\operatorname{Ker}\left\{\pi_{r+1}^{\prime}\left(\bar{d}_{0}\right): \pi_{r+1}^{\prime} \bar{B}_{1} \rightarrow \pi_{r+1}^{\prime} B_{0}\right\} \\
& \cong \operatorname{Ker}\{(i \otimes \mathbf{Z} / 2): G \otimes \mathbf{Z} / 2 \rightarrow F \otimes \mathbf{Z} / 2\} \cong \operatorname{Tor}\left(\pi_{r}^{\prime} Y, \mathbf{Z} / 2\right)
\end{aligned}
$$

as in $\S 5.2 .2$.

5.3.2. The inductive step. Now for each $k \geq r$, let $n=2(k-r)+1$, and assume $\bar{A}_{0}, \bar{A}_{1}, \ldots, \bar{A}_{n}$ and the $\bar{B}_{m}$ 's and $\bar{C}_{m}$ 's have been chosen so that (i)-(vi) of the proposition are satisfied for $0 \leq m \leq n$.

Note that (i) and (iv) imply that condition 4.4 .1(ii) of $\S 4.4$ holds-i.e., for each $n \geq 0$, we have $n \leq 2 \cdot\left(\left|\bar{A}_{n}\right|-r\right)+1$, so that Lemma 4.4 .3 applies. In fact, setting $Z \bar{B}_{n}=Z A_{n} \cap \bar{B}_{n}$, we have the following

5.3.3. Lemma. Assume that requirements 5.2.1(i) and (v) hold for $0 \leq m \leq$ $n=2(k-r)+1$; then $\pi_{k+1}^{\prime} Z A_{n} \cong \pi_{k+1}^{\prime} Z \bar{B}_{n} \oplus F$, where $F$ is a free Abelian group

5.3.4. Proof of Lemma 5.3.2. Since $r \geq 3$, we can use the argument of $\S 4.5 .4$ to show that

$$
\pi_{k+1}^{\prime} Z A_{n} \subseteq \pi_{k+1}^{\prime}\left(\bar{B}_{n} \amalg \bar{C}_{n}\right) \cong \pi_{k+1}^{\prime} \bar{B}_{n} \oplus \pi_{k+1}^{\prime} \bar{C}_{n} .
$$

Now let $\pi_{k+1}^{\prime} Z A_{n} \stackrel{i}{\hookrightarrow} \pi_{k+1}^{\prime}\left(\bar{B}_{n} \amalg \bar{C}_{n}\right) \stackrel{p}{\rightarrow} \pi_{k+1}^{\prime} \bar{C}_{n}$ be the inclusion and projection, respectively. Then we have a short exact sequence of Abelian groups

$$
0 \rightarrow \operatorname{Ker}(p \circ i) \rightarrow \pi_{k+1}^{\prime} Z A_{n} \rightarrow \operatorname{Im}(p \circ i) \rightarrow 0
$$

By 5.2.1(v) we have $\left|\bar{C}_{n}\right| \geq k+1$, so that $\pi_{k+1}^{\prime} \bar{C}_{n}$ is a free Abelian group. Thus $F=\operatorname{Im}(p \circ i)$ is free Abelian, too-and the sequence splits. Clearly $\pi_{k+1}^{\prime} Z \bar{B}_{n}=\operatorname{Ker}(p \circ i)$, so that $\pi_{k+1}^{\prime} Z A_{n} \cong \pi_{k+1}^{\prime} Z \bar{B}_{n} \oplus F$, as required. 
5.3.5. Completing the induction. For $n=2(k-r)+1 \geq 3$, we use assumptions 5.2.1(i) and (iv) to see that as in 5.3.1:

$$
\begin{aligned}
\pi_{k+1}^{\prime} Z \bar{B}_{n} & \cong \operatorname{Ker}\{(j \otimes \mathbf{Z} / 2): K \otimes \mathbf{Z} / 2 \rightarrow L \otimes \mathbf{Z} / 2\} \\
& \cong \operatorname{Tor}\left(\operatorname{Tor}\left(\pi_{r}^{\prime} Y, \mathbf{Z} / 2\right), \mathbf{Z} / 2\right) \cong \operatorname{Tor}\left(\pi_{r}^{\prime} Y, \mathbf{Z} / 2\right) .
\end{aligned}
$$

Lemmas 5.3.2 and 4.4.3 then allow us to choose $\bar{d}_{0}: \bar{B}_{n+2} \rightarrow \bar{B}_{n+1}$ satisfying 5.2.1(i) and (iv), as well as $\bar{C}_{n+1}$ and $\bar{C}_{n+2}$ satisfying 5.2.1(v) and (vi). This completes the proof of Proposition 5.2.1.

\section{Differentials in THE SPECTRAL SEQUENCE}

We now illustrate the fact that the differentials in the Hurewicz spectral sequence depend on higher-order homotopy information by using one to compute a certain Toda bracket for $\Sigma^{r-1} \mathbf{R} \mathbf{P}^{2}$.

In $\S 6.1$ we calculate the derived functors of $Q_{k}$ on the $\Pi$-algebra $\pi_{*} \Sigma^{r-1} \mathbf{R P}^{2}$ and find a nonvanishing differential in the $E^{2}$-term. In $\S 6.2$ we recall the construction of $V . \mathbf{X}$ and use it to give an explicit description of the differential in $\S 6.3$. Throughout this section $R=\mathbf{Z}$, and all homology is with integral coefficients.

6.1. A calculation for $\pi_{*} \Sigma^{r-1} \mathbf{R} \mathbf{P}^{2}$. Let $\mathbf{X}$ denote the $\mathbf{Z} / 2$-Moore space $\Sigma^{r-1} \mathbf{R} \mathbf{P}^{2}$, with $r \geq 4$. The first three nonvanishing rows in the $E^{2}$-term of the Hurewicz spectral sequence for $\mathbf{X}$ may then be calculated as follows:

6.1.1. П-algebra structure. The $\Pi$-algebra structure of $\pi_{*} \mathbf{X}$ in the first three nontrivial degrees in given by:

(i) $\pi_{r} \mathbf{X} \cong \mathbf{Z} / 2$, generated by an element $\alpha$;

(ii) $\pi_{r+1} \mathbf{X} \cong \mathbf{Z} / 2$, generated by $\alpha \circ \eta_{r}$;

(iii) $\pi_{r+2} \mathbf{X} \cong \mathbf{Z} / 4$, generated by an element $\beta$, with $2 \beta=\alpha \circ \eta_{r} \circ \eta_{r+1}$.

(This may be computed using [5, 6], for example).

6.1.2. Notation for free $\Pi$-algebras. Recall (§3.1.1) that $\boldsymbol{\Pi}$ denotes the homotopy category finite wedges of spheres, and let $\mathscr{F} \subset \Pi$-Alg denote the full subcategory of free $\Pi$-algebras (§3.1.2). The functor $\pi_{*}: \mathscr{T}_{*} \rightarrow \Pi$-Alg, when restricted to $\Pi$, induces an equivalence of categories between $\Pi$ and $\mathscr{F}$ (cf. $[10, I V, 4])$. We can thus describe free $\Pi$-algebras and their morphisms in terms of spheres and homotopy classes of maps between them.

In particular, we shall write $S^{k}=\pi_{*} \mathbf{S}^{k}$ for the free $\Pi$-algebra generated by a graded set having a single element in degree $k$. For $k \geq 3$ we let $\eta_{k}$ denote the generator of $\pi_{k+1}^{\prime} S^{k} \cong \pi_{k+1} \mathbf{S}^{k} \cong \mathbf{Z} / 2$, as well as the corresponding morphism $S^{k+1} \rightarrow S^{k}$.

6.1.3. A partial resolution. We are interested in the resolution of the $\Pi$-algebra $\pi_{*} \mathbf{X}$ only in degrees $\leq r+2$. In the above notation, we can partially describe a 
$C W$-basis for a resolution $A . \rightarrow Y$ as follows (omitting all "spheres" $S^{k}$ with $k>r+2$ ); subscripts indicate the simplicial dimension:

(i) $\bar{A}_{0}=S_{(0)}^{r} \amalg S_{(0)}^{r+2}$, with the augmentation $d_{0}: \bar{A}_{0} \rightarrow Y$ induced by $\alpha$ on $S_{(0)}^{r}$ and $\beta$ on $S_{(0)}^{r+2}$, respectively.

(ii) $\bar{A}_{1}=S_{(1)}^{r} \amalg S_{(1)}^{r+2}$. The attaching map $\bar{d}_{0}: \bar{A}_{1} \rightarrow A_{0}$, restricted to $S_{(1)}^{r}$, is a map of degree $2 S_{(1)}^{r} \rightarrow S_{(0)}^{r}$, while on $S_{(1)}^{r+2}$ it is the sum of a map of degree $2 S_{(1)}^{r+2} \rightarrow S_{(0)}^{r+2}$ and the map $\eta_{r} \circ \eta_{r+1}: S_{(1)}^{r+2} \rightarrow S_{(0)}^{r}$.

(iii) $\bar{A}_{2}=S_{(2)}^{r+1}$, with $\bar{d}_{0}: \bar{A}_{2} \rightarrow A_{1}$ given by $\eta_{r}: S_{(r)}^{r+1} \rightarrow S_{(1)}^{r}$.

(iv) $\bar{A}_{3}=S_{(3)}^{r+1}$, with $\bar{d}_{0}: \bar{A}_{3} \rightarrow A_{2}$ given by a map of degree $2 S_{(3)}^{r+1} \rightarrow S_{(2)}^{r+1}$.

(v) $\bar{A}_{n}=0$ in degrees $\leq r+2 \quad n \geq 4$.

6.1.4. The $E^{2}$-term. Using this resolution, it is easy to calculate the first three nonvanishing rows of $E_{n, k}^{2} \cong\left(L_{n} Q_{k}\right) \pi_{*} \mathbf{X}$ for the Hurewicz spectral sequence of $\mathbf{X}$, as follows:

\begin{tabular}{r|ccccc}
$r+2$ & $\mathbf{Z} / 2$ & 0 & 0 & 0 & $\cdots$ \\
$r+1$ & 0 & 0 & $\mathbf{Z} / 2$ & 0 & $\cdots$ \\
$r$ & $\mathbf{Z} / 2$ & 0 & 0 & 0 & $\cdots$ \\
\hline$k$ & 0 & 1 & 2 & 3 & $\cdots$
\end{tabular}

6.1.5. A differential. The spectral sequence converges to the reduced integral homology of the Moore space $\mathbf{X}$, where $\widetilde{H}_{r} \mathbf{X} \cong \mathbf{Z} / 2$ and $\widetilde{H}_{i} \mathbf{X}=0$ for $i \neq r$. Therefore, the differential $d^{2}: E_{2, r+1}^{2} \rightarrow E_{0, r+2}^{2}$ is necessarily nontrivial (and so an isomorphism).

6.1.6. $E_{0, r+2}^{2}$. By $\S 2.2 .1$, we know that the nonzero element of $E_{0, r+2}^{2} \cong \mathbf{Z} / 2$ may be represented by the generator $\beta \in \pi_{r+2} \mathbf{X}$, modulo the subgroup of $\pi_{r+2} \mathbf{X}$ generated by the single "composable" $\alpha \circ \eta_{r} \circ \eta_{r+1}=2 \cdot \beta$, so that the indeterminacy is just in the choice of the generator $\beta$ for $\mathbf{Z} / 4 \cong \pi_{r+2} \mathbf{X}$.

6.2. The simplicial space $V . \mathbf{X}$. We now recall the construction of $V . \mathbf{X}$ from $[18, \S 2]$, and introduce some notation:

6.2.1. The cotriple $\mathscr{V} . V . \mathbf{X}$ is constructed by means of a cotriple $\mathscr{V}: \mathscr{T}_{*} \rightarrow$ $\mathscr{T}_{*}$, which assigns to each pointed connected space $\mathbf{X} \in \mathscr{T}_{*}$ the space

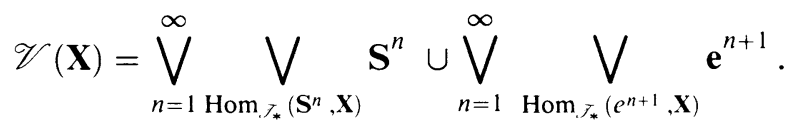

For each $n \geq 1$, choose a fixed homeomorphism $\phi: \partial\left(\mathbf{e}^{n+1}\right) \cong \mathbf{S}^{n}$ between the boundary of the $(n+1)$-disc and the $n$-sphere. The $(n+1)$-disc indexed by $F: \mathbf{e}^{n+1} \rightarrow \mathbf{X}$ is then attached by $\phi$ to the $n$-sphere $\mathbf{S}^{n}$ indexed by $f=$ $\left.F\right|_{\partial\left(\mathbf{e}^{n+1}\right)}$. 
The counit of $\mathscr{V}$, a natural transformation $\varepsilon: \mathscr{V} \rightarrow i d_{\mathscr{T}_{*}}$, is given by the "evaluation on the index" map $\varepsilon_{\mathbf{X}}: \mathscr{V}(\mathbf{X}) \rightarrow \mathbf{X}$. This maps the sphere $\mathbf{S}^{n} \subset$ $\mathscr{V}(\mathbf{X})$ indexed by $f \in \operatorname{Hom}_{\mathscr{T}_{*}}\left(\mathbf{S}^{n}, \mathbf{X}\right)$ into the space $\mathbf{X}$ by the map $f$, and similarly for discs.

6.2.2. $V . \mathbf{X}$. The simplicial space $V . \mathbf{X}$ is then defined by setting $V_{n} \mathbf{X}=$ $\mathscr{V}^{n+1} \mathbf{X}$, with the usual face and degeneracy maps induced by the counit and comultiplication of the cotriple (cf. [2, I, §4.1]).

To avoid confusion, the face and degeneracy maps of $V . \mathbf{X}$ will be considered to be "horizontal" (by analogy with the bisimplicial set $S V . \mathbf{X}$ ) and will be marked with a superscript $h$ (e.g., $d_{j}^{h}$ ).

6.2.3. Notation for $V . \mathbf{X}$. To describe some of the spheres of $V . \mathbf{X}$, we can use the following notation:

For any map $f \in \operatorname{Hom}_{\mathcal{T}_{*}}\left(\mathbf{S}^{n}, \mathbf{X}\right)$, we denote the $n$-sphere in $V_{0} \mathbf{X}$ indexed by $f$ by $\mathbf{S}_{(f)}^{n}$. Similarly, if $F \in \operatorname{Hom}_{\mathscr{T}_{*}}\left(\mathbf{e}^{n+1}, \mathbf{X}\right)$ is a nullhomotopy of $f$, we denote the corresponding $(n+1)$-disc in $V_{0} \mathbf{X}$ by $\mathbf{e}_{(F)}^{n+1}$, so that $\mathbf{S}_{(f)}^{n} \cup \mathbf{e}_{(F)}^{n+1} \subset V_{0} \mathbf{X}$.

In certain special cases, we can also use such a notation for the spheres of $V_{n} \mathbf{X}$ $(n \geq 1)$ : if the map $g: \mathbf{S}^{m} \rightarrow V_{0} \mathbf{X}$ factors through the inclusion $\mathbf{S}_{(f)}^{n} \hookrightarrow V_{0} \mathbf{X}$, we denote the $m$-sphere of $V_{1} \mathbf{X}$ corresponding to $g$ by a double index: $\mathbf{S}_{(f, g)}^{m}$, and so on.

In such cases, the face maps of $V . \mathbf{X}$ have a particularly simple description. For example, the face map $d_{0}^{h}: V_{1} \mathbf{X} \rightarrow V_{0} \mathbf{X}$ on $\mathbf{S}_{(f, g)}^{n}$ is just $f: \mathbf{S}_{(f, g)}^{n} \rightarrow$ $\mathbf{S}_{(g)}^{m} \hookrightarrow V_{0} \mathbf{X}$, while $d_{1}^{h}$ is given by a homeomorphism $\mathbf{S}_{(f, g)}^{n} \stackrel{\cong}{\rightrightarrows} \mathbf{S}_{(f \circ g)}^{n} \hookrightarrow V_{0} \mathbf{X}$. Other face maps on such spheres are also included by suitable "compositions of indices."

6.3. The differential $d^{2}$. We now want to understand the nontrivial differential of $\S 6.15$ :

6.3.1. The double complex. Recall from $\S 2.1 .3$ that our spectral sequence is the Quillen spectral sequence of the bisimplicial Abelian group $\mathbf{Z} S V$.X. If $K$ is the functor which takes a simplicial Abelian group to its associated chain complex [11, p. 235], then applying $K$ twice to $\mathbf{Z} S V . \mathbf{X}$ yields a double chain complex $C_{. .}=K(\mathbf{Z} S V . \mathbf{X})$, and our spectral sequence is isomorphic to the usual spectral sequence of this double complex [3, Chapter XV, §6], with $E_{n, k}^{2} \cong H_{n}^{h} H_{k}^{v} C_{.,}$.

6.3.2. $E_{2, r+1}^{0}$. In the notation of $\S 6.2 .3$, we have the following spheres in $V . \mathbf{X}$ :

Choose some map $a: \mathbf{S}^{r} \rightarrow \mathbf{X}$ representing the element $\alpha \in \pi_{r}(\mathbf{X})$; as above, we denote by $\mathbf{S}_{(a)}^{r}$ the corresponding $r$-sphere in $V_{0} \mathbf{X}$. Similarly, let $\mathbf{S}_{(a, t)} \subset$ $V_{1} \mathbf{X}$ correspond to a map $t: \mathbf{S}^{r} \rightarrow \mathbf{S}_{(a)}^{r} \hookrightarrow V_{0} \mathbf{X}$ of degree 2, and let $\mathbf{S}_{(a, t, h)}^{r+1} \subset V_{2} \mathbf{X}$ correspond to a map $h: \mathbf{S}^{r+1} \rightarrow \mathbf{S}_{(a, t)}^{r+1} \hookrightarrow V_{1} \mathbf{X}$ representing $\eta_{r} \in \pi_{r+1} \mathbf{S}_{(a, t)}^{r}$. 
The generator of $H_{2}^{h} H_{r+1}^{v} C_{\text {.. }} \cong E_{2, r+1}^{2} \cong \mathbf{Z} / 2$ may then be represented by the element $1 \cdot \sigma \in C_{2, r+1} \cong E_{2, r+1}^{0}$, where $\sigma \in S_{r+1} \mathbf{S}_{(a, t, h)}^{r+1}$ is a singular $(r+1)$ simplex representing a generator of $\widetilde{H}_{r+1} \mathbf{S}_{(a, t, h)}^{r+1}$.

6.3.3. A diagram chase. With this description, we need only the usual diagram chase to calculate the differential of $\S 6.1 .5$ :

(i) The horizontal differential $\partial^{h}: H_{r+1}^{v} C_{2,} \rightarrow H_{r+1}^{v} C_{1}$. is given as the alternating sum of the horizontal face maps, so that by $\S 6.2 .3$ we have:

$$
\partial^{h}\langle 1 \cdot \sigma\rangle=\left\langle d_{0}^{h}(\sigma)-d_{1}^{h}(\sigma)+d_{2}^{h}(\sigma)\right\rangle=\left\langle h_{*}(\sigma)\right\rangle-\left\langle\sigma^{\prime}\right\rangle+\left\langle\sigma^{\prime \prime}\right\rangle,
$$

with $\sigma^{\prime} \in S_{r+1} \mathbf{S}_{(a \circ t, h)}^{r+1}$ and $\sigma^{\prime \prime} \in S_{r+1} \mathbf{S}_{(a, t o h)}^{r+1}$ being singular $(r+1)$-simplices representing generators in homology, and $h_{*}(\sigma) \in S_{r+1} \mathbf{S}_{(a, t)}^{r}$ a singular $(r+1)$ simplex representing the generator $\eta_{r} \in \pi_{r+1} \mathbf{S}_{(a, t)}^{r}$.

(ii) Since $\widetilde{H}_{r+1} \mathbf{S}_{(a, t)}^{r}=0$, the cycle $h_{*}(\sigma) \in \mathbf{Z} S_{r+1} \mathbf{S}_{(a, t)}^{r}$ has a singular $(r+2)$ chain $\gamma \in \mathbf{Z} S_{r+2} \mathbf{S}_{(a, t)}^{r}$ with $\partial^{v}(\gamma)=h_{*}(\sigma)$.

Furthermore, $a \circ t: \mathbf{S}_{(a \circ t)}^{r} \rightarrow \mathbf{X}$ (representing $2 \alpha \in \pi_{r} \mathbf{X}$ ) is nullhomotopic, so that the sphere $\mathbf{S}_{(a \circ t)}^{r} \subset V_{0} \mathbf{X}$ indexed by $a \circ t$ has various $(r+1)$-discs attached to it, corresponding to all possible nullhomotopies of $a \circ t$ : let $F: \mathrm{e}^{r+1} \rightarrow \mathbf{X}$ be one such nullhomotopy, and $\mathbf{e}_{(F)}^{r+1} \subset V_{0} \mathbf{X}$ the corresponding $(r+1)$-disc.

We can therefore extend the map $h: \mathbf{S}_{(a \circ t, h)}^{r+1} \rightarrow \mathbf{S}_{(a \circ t)}^{r}$ to a map $C h: \mathbf{e}_{(F, C h)}^{r+2} \rightarrow$ $\mathbf{e}_{(F)}^{r+1}$, where $\mathbf{e}_{(F, C h)}^{r+2} \subset V_{1} \mathbf{X}$ is attached to $\mathbf{S}_{(a \circ t, h)}^{r+1}$. In particular, we have a singular $(r+2)$-simplex $\zeta \in S_{r+2} \mathbf{e}_{(F, C h)}^{r+2}$ with $\partial^{v}(1 \cdot \zeta)=\sigma^{\prime}$, which represents a generator of $\tilde{H}_{r+2}\left(\mathbf{e}_{(F, C h)}^{r+2}, \mathbf{S}_{(a \circ t, h)}^{r+1}\right)$.

Similarly, since $[t \circ h]=2 \eta_{r}=0 \in \pi_{r+1} \mathbf{S}^{r}$, we can choose a nullhomotopy $G: \mathbf{e}^{r+1} \rightarrow \mathbf{S}_{(a)}^{r}$ for $t \circ h$; let $\mathbf{e}_{(a, G)}^{r+2} \subset V_{1} \mathbf{X}$ be the corresponding $(r+2)$-disc. We have a singular $(r+2)$-simplex $\zeta^{\prime} \in S_{r+2} \mathbf{e}_{(a, G)}^{r+2}$ with $\partial^{v}\left(\zeta^{\prime}\right)=\sigma^{\prime \prime}$. Thus

$$
\partial^{\prime \prime}\left\langle\gamma-\zeta+\zeta^{\prime}\right\rangle=\left\langle h_{*}(\sigma)-\sigma^{\prime}+\sigma^{\prime \prime}\right\rangle=\partial^{h}\langle 1 \cdot \sigma\rangle \in H_{r+1}^{v} C_{1},
$$

(iii) Finally, $d^{2}\langle\sigma\rangle \in E_{2, r+2}^{2}$ is represented in

$$
C_{0, r+2} \cong E_{2, r+2}^{0}
$$

by $\partial^{h}\left\langle\gamma-\zeta+\zeta^{\prime}\right\rangle$, where $\partial^{h}=d_{0}^{h}-d_{1}^{h}$.

By $\S 6.2 .3$ we have $\partial^{h}(\gamma)=t_{*}(\gamma)-\gamma^{\prime}$; but neither of the $(r+2)$-chains $t_{*}(\gamma) \in \mathbf{Z} S_{r+2} \mathbf{S}_{(a)}^{r}$ and $\gamma^{\prime} \in \mathbf{Z} S_{r+2} \mathbf{S}_{(a \circ t)}^{r}$ contributes anything to $\widetilde{H}_{r+2} V_{0} \mathbf{X}$, for dimensional reasons.

Also, $\partial^{h}(\zeta)=(C h)_{*}(\zeta)-\xi$, where $(C h)_{*}(\zeta) \in S_{r+2} \mathbf{S}_{(a \circ t)}^{r}$ again contributes nothing to $\widetilde{H}_{r+2}\left(V_{0} \mathbf{X}\right)$, and $\xi \in S_{r+2} \mathbf{e}_{(F \circ C h)}^{r+2}$ represents a generator of $\widetilde{H}_{r+2}\left(\mathbf{e}_{(F \circ C h)}^{r+2}, \mathbf{S}_{(a \circ 1 \circ h)}^{r+1}\right)$. 
Similarly, $\partial^{h}\left(\zeta^{\prime}\right)=G_{*}\left(\zeta^{\prime}\right)-\xi^{\prime}$, with $G_{*}\left(\zeta^{\prime}\right)$ in $S_{r+2} \mathbf{S}_{(a)}^{r}$ contributing nothing to $\widetilde{H}_{r+2} V_{0} \mathbf{X}$, and $\xi^{\prime} \in S_{r+2} \mathbf{e}_{(a \circ G)}^{r+2}$ again a generator of $\widetilde{H}_{r+2}\left(\mathbf{e}_{(a \circ G)}^{r+2}, \mathbf{S}_{(a \circ t \circ h)}^{r+1}\right)$.

Now, since the two $(r+2)$-cells $\mathbf{e}_{(F \circ C h)}^{r+2}$ and $\mathbf{e}_{(a \circ G)}^{r+2}$ are both attached to the same $(r+1)$-sphere $\mathbf{S}_{(a \circ t \circ h)}^{r+1} \subset V_{0} \mathbf{X}$, they together form an $(r+2)$-sphere $\mathbf{S}_{(b)}^{r+2} \subset V_{0} \mathbf{X}$ (though not one of those explicitly included in the definition of $V_{0} \mathbf{X}=\mathscr{V}(\mathbf{X})$ in $\left.\S 6.2 .1\right)$. Here $b: \mathbf{S}^{r+2} \rightarrow \mathbf{X}$ represents the element in the Toda bracket

$$
[b] \in\left\langle\alpha, 2, \eta_{r}\right\rangle \subset \pi_{r+2} \mathbf{X}
$$

which is determined by the maps $a, t, h$ (representing the homotopy classes $\alpha, 2, \eta_{r}$ respectively) and the nullhomotopies $F: a \circ t \sim 0, G: t \circ h \sim 0$.

Thus the part of $\partial^{h}\left\langle\gamma-\zeta+\zeta^{\prime}\right\rangle$ which does not vanish in $\widetilde{H}_{r+2} V_{0} \mathbf{X}$ represents a homology generator $\left\langle\sigma_{(b)}\right\rangle \in \widetilde{H}_{r+2} \mathbf{S}_{(b)}^{r+2}$, so in $E_{0, r+2}^{1} \cong \widetilde{H}_{r+2} V_{0} \mathbf{X}$ we have

$$
\left\langle\partial^{h}\left(\gamma-\zeta+\zeta^{\prime}\right)\right\rangle=\left\langle\xi-\xi^{\prime}\right\rangle=\left\langle\sigma_{(b)}\right\rangle \in \widetilde{H}_{r+2} V_{0} \mathbf{X} .
$$

Since we know that $d^{2}\langle\sigma\rangle=\langle\beta\rangle \in E_{0, r+2}^{2}$ (\$6.1.5), we conclude that the generator $\beta$ of $\pi_{r+2} \Sigma^{r-1} \mathbf{R} \mathbf{P}^{2} \cong \mathbf{Z} / 4$ is in the Toda bracket $\left\langle\alpha, 2, \eta_{r}\right\rangle$ (with the same indeterminacy as in the choice of generator for $Z / 4$, by $\S 6.1 .6$ ).

\section{Related SPECTRAL SEQUeNCES}

We now note the existence of two related spectral sequences: one for any generalized homology theory $(\S 7.1)$ and one a "Kunneth spectral sequence" for the smash of two spectra $(\S 7.2)$.

7.1. Generalized homology theories. First, we can generalize the Hurewicz spectral sequence for ordinary homology $H_{*}$ to any reduced generalized homology theory $k_{*}$, to obtain

7.1.1. A generalized Hurewicz spectral sequence. Let $\mathbf{X} \in \mathscr{T}_{*}$ be a pointed connected space, and $V . \mathbf{X}$ the augmented simplicial space of $\S 2.1 .1$, with $\Delta V . \mathbf{X} \simeq$ $\mathbf{X}$. The realization $\Delta V . \mathbf{X}$ has a filtration by subcomplexes $\Delta^{0} V . \mathbf{X} \subset \Delta^{1} V . \mathbf{X} \cdots \subset$ $\Delta V . \mathbf{X}$, where $\Delta^{n} V . \mathbf{X}$ is the realization of the $n$-skeleton of the simplicial space $V . \mathbf{X}$ (cf. $[17, \S 5])$.

Applying the generalized homology functor $k_{*}$ to this filtration, we obtain a spectral sequence abutting to $k_{*} \Delta V . \mathbf{X} \cong k_{*} \mathbf{X}$, with $E_{r, s}^{2}=\pi_{r} k_{2} V . \mathbf{X}$, as in [17, Proposition 5.1]. The spectral sequence converges strongly to $k_{*} \mathbf{X}$ if $k_{*}$ is connective.

7.1.2. The $E^{2}$-term as derived functors. Recall from $\S 6.1 .2$ that the functor $\pi_{*}: \Pi \rightarrow \mathscr{F}$ induces an equivalence of categories between $\Pi$, the homotopy category of finite wedges of spheres, and $\mathscr{F} \subset \Pi$-Alg, the full subcategory of free $\Pi$-algebras. Thus, we can describe a functor on $\mathscr{F}$ (noncanonically) by 
specifying it on $\Pi$. Moreover, we only need a functor defined on $\mathscr{F}$ in order for the derived functors to be defined for all $\Pi$-algebras, by $\S 3.2 .4$.

In particular, for any generalized homology theory $k_{*}$ each of the functors $k_{j}: h o \mathscr{T}_{*} \rightarrow A b g p$ defines a functor $\tilde{k}_{j}: \mathscr{F} \rightarrow A b g p$; as in $\S 3.3$, the $E^{2}$-term of the above generalized Hurewicz spectral sequence may be described in terms of derived functors:

$$
E_{n, j}^{2}=\pi_{n} k_{j} V . \mathbf{X} \cong L_{n}\left(\tilde{k}_{j}\right) \pi_{*} \mathbf{X} .
$$

Thus, if $k_{*}$ is connective, the proof of Theorem 4.1 carries over to yield a similar vanishing line for the $E^{2}$-term. If the coefficients $k_{*}(p t)$ are torsion free, Corollary 4.1.1(ii) also applies.

7.2. A stable spectral sequence. It should be pointed out that a stable version of the Hurewicz spectral sequence has long been known: in [9, Theorem 4.8], T. Y. Lin describes a Künneth spectral sequence for the smash of two spectra, as follows:

Let $\boldsymbol{\pi}_{*}$ denote the stable homotopy ring of spheres: $\boldsymbol{\pi}_{*}=\pi_{*} \mathbf{S}^{0}$, and let $X, Y$ be two spectra, with $Y$ connected. Then there is a spectral sequence converging to $\pi_{*}(X \wedge Y)$, with $E_{r, s}^{2} \cong \operatorname{Tor}^{\pi_{*}}\left(\pi_{*} X, \pi_{*} Y\right)$ (cf. [1, p. 7]). Thus, if $Y$ is a spectrum corresponding to a connective homology theory $k_{*}$, this gives us a spectral sequence converging to $k_{*} X$, whose $E^{2}$-term depends only on $k_{*}$ and the $\pi_{*}$-module structure of $\pi_{*} X$.

\section{REFERENCES}

1. J. F. Adams, Lectures on generalized cohomology, Category Theory, Homology Theory and Their Applications III, Lecture Notes in Math., vol. 99, Springer-Verlag, Berlin and New York, 1969.

2. A. K. Bousfield and D. M. Kan, Homotopy limits, completions, and localizations, Lecture Notes in Math., vol. 304, Springer-Verlag, Berlin and New York, 1972.

3. H. Cartan and S. Eilenberg, Homological algebra, Princeton Univ. Press, Princeton, N.J., 1956.

4. P. J. Hilton, On the homotopy groups of the union of spheres, J. London Math. Soc. 30 (1955), 154-172.

5. __ Calculating the homotopy groups of $A_{n}^{2}$-polyhedra. I, Quart. J. Math. Oxford Ser. (2) 1 (1950), 299-309.

6. __ Calculating the homotopy groups of $A_{n}^{2}$-polyhedra. II, Quart. J. Math. Oxford Ser. (2) 1 (1951), 228-240.

7. D. M. Kan, Minimal free c.s.s. groups, Illinois J. Math. 2 (1958), 537-547.

8. $\ldots$, A relation between $C W$-complexes and free c.s.s. groups, Amer. J. Math. 81 (1959), 512-528.

9. T. Y. Lin, Homological algebra of the stable homotopy ring $\pi_{*}$ of spheres, Pacific J. Math. 38 (1971), 117-143.

10. S. Mac Lane, Categories for the working mathematician, Springer-Verlag, Berlin and New York, 1971.

11. ___ Homology, Springer-Verlag, Berlin and New York, 1963.

12. J. P. May, Simplicial objects in algebraic topology, Univ. of Chicago, Chicago, London, 1967.

13. J. C. Moore, Homotopie des complexes monoïdaux, I, Sèm. Henri Cartan 7 (1954-55), §18. 
14. D. G. Quillen, Homotopical algebra, Lecture Notes in Math., vol. 43, Springer-Verlag, Berlin and New York, 1967.

15. __, On the (co-)homology of commutative rings, Proc. Sympos. Pure Math., vol. 17, Amer. Math. Soc., Providence, R.I., 1970, pp. 65-87.

16. __ Spectral sequences of a double semi-simplicial group, Topology 5 (1966), 155-156.

17. G. Segal, Classifying spaces and spectral sequences, Inst. Hautes Ètudes Sci. Publ. Math. 34 (1968), 105-112.

18. C. Stover, A Van Kampen spectral sequence for higher homotopy groups, Ph.D. thesis, Massachusetts Institute of Technology, 1988; Topology (to appear).

19. G. W. Whitehead, Elements of homotopy theory, Springer-Verlag, Berlin and New York, 1971.

Department of Mathematics, Massachusetts Institute of Technology, Cambridge, MASSACHUSETTS 02139

Current address: Department of Mathematics, Northwestern University, Evanston, Illinois 60208 